\title{
Combined sources of motivation and escape responding'
}

Zero and 21/4 hr. (approximately) of food deprivation were orthogonally combined with 40 and $70 v$ of shock during a single shock-escape trial. Escape performance was directly related to both level of deprivation and shock intensity.

Drive theories of primary motivation (e.g., Spence, 1956) assume that, all things equal, the more numerous the primary sources of drive, the greater the motivation, and the more vigorous the response. Most studies of combined sources of primary motivation have investigated appetitional motive combinations (i.e., food deprivation combined with water deprivation). Only Amsel (1950) has reported on the combining of aversive and appetitional motives. This investigator varied shock intensity (aversive motive) and food deprivation during escape training but found no reliable effects of combined motivation.

The present experiment reports on combining primary aversive and appetitional motives during a single shock-escape trial. A single trial technique was used as one way to avoid the confounding between motivational and reinforcing aspects of shock, which occurred in Amsel's study. Amsel trained Ss to escape from different shock intensities to no shock. Thus, Ss differed in level of shock-produced motivation and in level of reinforcement due to amount of shock offset. The present study assumed that escape performance on a single (and only) shock trial was independent of previous (non-existent) shock reinforcements and should reflect only motivational factors.

Method

Apparatus. The apparatus was that described by Franchina (1966). Briefly, a white start box was separated from a black safe box by a guillotine door and a hurdle. The start box floor could be electrified from a constant voltage source. The safe box floor was masonite and could be depressed. The lid of each box contained light sources which maintained constant levels of illumination in the start and safe boxes. The start box lid also contained a light source for discrete CS presentations in that box.

Subjects, Design, and Procedures. Ss were 56 naive, male, Holtzman, albino rats. At 98-101 days old, Ss were weighed and started on a feeding schedule of $1-1 / 2 \mathrm{hr}$. access to food (ad lib) every $24 \mathrm{hr}$. Water was always available.

After eight days on this maintenance schedule, each $S$ received two successive days of exploration in the apparatus and, then, two successive days of one trial of hurdle-jumping per day. Ss were weighed on each hurdle-jumping day, $30 \mathrm{~min}$. before their daily feeding.
For each S, an exploration day consisted of two $45 \mathrm{sec}$. periods in each side of the apparatus, with the guillotine door closed. A daily hurdle-jumping trial began with the insertion of $S$ into the start box. Ten sec. later, the guillotine door was raised, simultaneously activating the CS and a .01 sec. timer. If $S$ jumped the hurdle, the safe box floor depressed, terminating CS and timer. If $S$ failed to jump within 40 sec. $S$ was removed from the start box and a latency of 40 sec. was recorded. The first hurdle-jumping trial was administered without shock to provide a baseline index of hurdle-jumping to the start box cues alone. On the second trial of hurdle-jumping, half of the Ss received $40 \mathrm{v}$ shock, half, $70 \mathrm{v}$ shock, presented simultaneously with the raising of the guillotine door. Since all Ss had the same feeding schedule, appetitional motivation was manipulated at hurdle-jumping time in terms of hours since Ss' last daily feeding. Half of the Ss received each hurdle-jumping trial from 21-1/4 to $21-3 / 4 \mathrm{hr}$. after the previous daily feeding and were fed a minimal $45 \mathrm{~min}$. after the daily trial. The other half of the Ss received each hurdle-jumping trial from $1 / 12$ to $1 / 2 \mathrm{hr}$. after a daily feeding.

Thus this experiment combined appetitional motive sources (0- or 21-1/4 hr. approximately of food deprivation) with aversive motive sources ( 40 or $70 \mathrm{v}$ shock) in a 2 by 2 design with 14 Ss randomly assigned per cell.

Results

Results of separate factorial analyses of the Ss' weights at the start of deprivation and on each day of hurdle-jumping showed that no reliable weight differences existed prior to deprivation nor on the days of hurdle-jumping.

Figure 1 shows that hurdle-jumping performance was relatively uniform on the non-shock trial but was directly related to both intensity of shock and level of food deprivation on the shock trial. A trend analysis of the overall data of Fig. 1 yielded significant $F$-values for Shock Intensity $(F=22.68, p<.001)$, Trials $(F=245.72, p<.001)$, Trials by Shock Intensity $(F=41.77, p<.001)$, and Trials by Deprivation $(F=7.76$, $p<.01)$. The of were 1 and 52 throughout. The significant interactions were explained with factorial analyses which yielded significant F-values for Shock Intensity and Deprivation on the shock trial ( $F=38.67,7.72$, $\mathrm{df}=1 / 52, \mathrm{p}<.001,<.01$, respectively) but not on the non-shock trial.

A reciprocal transformation of the latency data showed essentially the relationships of Fig. 1. An overall trend analysis of these data yielded significant 


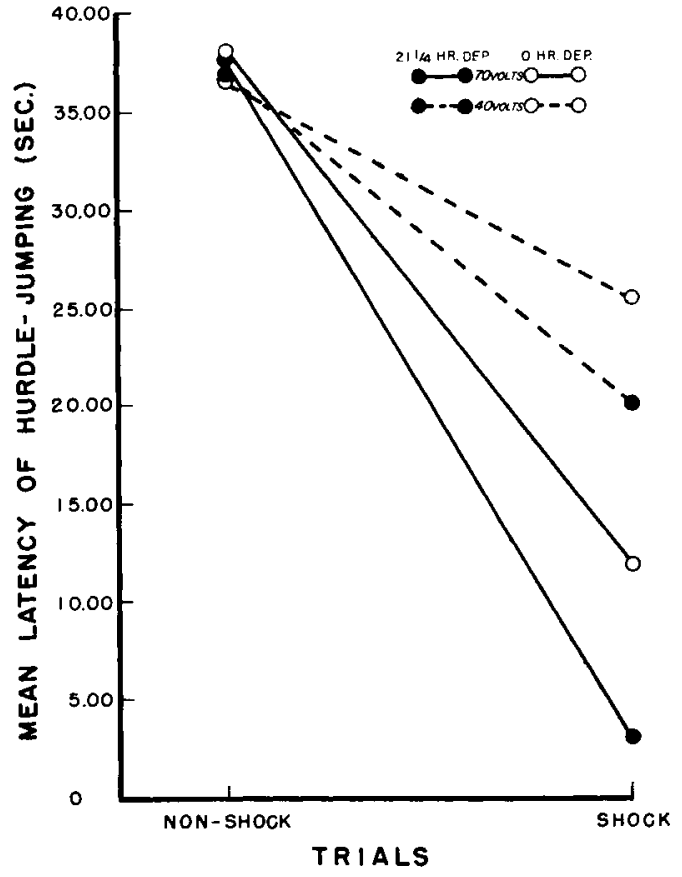

Fig. 1. Mean latency of hurdle-jumping on non-shock and shock trials for groups that received 40 - or $70-\mathrm{v}$ shock under 0 - or $21 \frac{1}{4}-\mathrm{hr}$. food deprivation.

F-values for Shock Intensity $(F=20.77, p<.001)$, Deprivation $(F=10.77, \mathrm{p}<.005)$, Trials $(F=33.85, \mathrm{p}<$ $.001)$, and their interaction, Trials by Shock Intensity by Deprivation $(F=7.69, p<.01)$. The df were 1 and 52 throughout. Evaluation of the significant triple interaction showed that the factors Shock Intensity, Deprivation and the Shock Intensity by Deprivation interaction were significant on the shock trial $(F=21.80,10.00$, $6.80, \mathrm{df}=1 / 52, \mathrm{p}<.001,<.005,<.025$, respectively); while no factors were significant on the non-shock trial. The interaction between Shock Intensity and Deprivation in the shock trial analysis was due to a non-significant difference between the deprivation groups in the $40-v$ condition $(t<1)$. The difference between the deprivation groups in the $70 \mathrm{v}$ condition was significant $(t=4.38, p<.001)$ as were the differences between the shock groups in the $0-$ and in the $21-1 / 4-h r$. deprivation conditions $(t=2.12,5.50, p<.05$, $<.001$, respectively). The df were 52 throughout. Discussion

This study showed that combining shock intensity and food deprivation tended to augment the strength of an unconditioned (i.e., reflexively-elicited, untrained) escape-from-shock response. These results were consistent, first, with drive theory assumptions about combined motives, second, with the results of learning experiments on combined appetitional motives (e.g., Kendler, 1945) and, third, with the data concerning unconditioned response strength under primary appetitional and secondary aversive motive combinations (Meryman, 1952).

The data also showed that the relationship between shock intensity and food deprivation as combined motives was mitigated by the properties of the measurement scales used. Analysis of the latency data showed that deprivation levels produced significant differences at each shock level, and vice versa. Analysis of the reciprocal of latency data showed that deprivation levels produced significant differences at the high shock level but not at the low shock level. The interaction of shock intensity and deprivation, observed in the latter analysis, may have been due to the tendency of the reciprocal scale to augment the differences between scores at high levels of response strength (i.e., short latency scores) but to diminish the differences between scores at low levels of response strength (i.e., long latency scores). Since the hurdle-jumping response in the present study was presumed to be elicited weakly on the first 40-v shock trial, long latencies occurred for both deprivation groups. The reciprocal transformations of these data may have tended to obscure the performance differences by assigning similar reciprocal values to quite different latency scores (e.g., the latencies of $22 \mathrm{sec}$. and $40 \mathrm{sec}$. have reciprocals of only .04 and .02, respectively, while their latency difference is 18 sec.).

\section{References}

Amsel, A. The combination of a primary appetitional need with primary and secondary emotionally derived needs. $J$. exp. Psychol., 1950, 40, 1-14.

Franchina, J. J. Effects of shock schedules on the acquisition and extinction of escape behavior. Psychon. Sci., 1966, 4, 277-278.

Kendler, H. H. Drive interaction: 1. Learning as a function of the simultaneous presence of hunger and thirst drives, $J$. exp. Psychol., 1945, 35, 96-109.

Meryman, J. J. Magnitude of startle response as a function of hunger and fear. Unpublished master's thesis, State University of Iowa, 1952.

Spence, K. W. Behavior theory and conditioning. New Haven: Yale University Press, 1956.

\section{Note}

1. Supported by Grant No. 20-97-152 from the Research Institute of Southern Methodist University. 